\title{
Understanding Wavelength Scaling in 19-Cell Core Hollow-Core Photonic Bandgap Fibers
}

\author{
Y. Chen, N. V. Wheeler, N. K. Baddela, J. R. Hayes, S. R. Sandoghchi, E. Numkam Fokoua, M. Li, \\ F. Poletti, M. N. Petrovich, and D. J. Richardson \\ Optoelectronics Research Centre, University of Southampton, Southampton SO17 1BJ, United Kingdom \\ yc1m12@soton.ac.uk
}

\begin{abstract}
First experimental wavelength scaling in 19-cell core HC-PBGF indicates that the minimum loss waveband occurs at longer wavelengths than previously predicted. Record low loss $(2.5 \mathrm{~dB} / \mathrm{km})$ fibers operating around $2 \mu \mathrm{m}$ and gas-purging experiments are also reported. OCIS codes: (060.5295) Photonic crystal fibers; (060.2330) Fiber optics communications
\end{abstract}

\section{Introduction}

Hollow-core photonic bandgap fibers (HC-PBGFs) have demonstrated potential to address some of the intrinsic capacity limitations of standard transmission fibers while providing a substantial reduction in signal latency [1]. Scattering from thermodynamically generated surface roughness has been identified as the underlying source of loss for these fibers and a number of theoretical studies [2,3] predict a $\sim \lambda^{-3}$ wavelength dependence and identify the waveband at $\sim 1.9 \mu \mathrm{m}$ as the region beyond which material absorption becomes the dominant loss mechanism. The wavelength of minimum predicted loss is thus determined by the amount of fractional overlap of the guided mode with the fiber structure, which affects both loss mechanisms [2]. Experimental support for these predictions has been provided by loss studies on 7 cell HC-PBGFs [2,4], however no detailed investigation has been reported for larger core, 19 cell HC-PBGFs, which can achieve lower loss and thus have more practical value for telecoms applications. Moreover it is important to identify what drawing parameters contribute to determine the wavelength of minimum loss in real fibers and verify how effective is wavelength scaling to reduce the loss of these fibers.

Here we report to the best of our knowledge the first experimental wavelength scaling study in 19 cell HCPBGFs. By measuring the transmission of several homothetically scaled fibers we confirm the validity of the $\sim \lambda^{-3}$ dependence of the scattering loss, and demonstrate that the wavelength of minimum loss occurs at longer wavelengths (around $2.1 \mu \mathrm{m}$ ) than previously predicted [2]. A $\sim 2.5 \mathrm{x}$ loss reduction is achieved by shifting the operating wavelength from $1.5 \mu \mathrm{m}$ to $2.1 \mu \mathrm{m}$. Our measurements also seem to indicate that the wavelength of minimum loss is influenced by material properties in addition to mode field overlap. Finally, we demonstrate that undesired gas absorptions can be removed by purging fabricated fibers even in multi-hundred meter lengths.

\section{Fiber fabrication and results}

The fibers utilized in this study had a 19 cell core and were fabricated using a two-step stack and draw technique. The primary stack had $7 \frac{1}{2}$ rings of capillaries constituting the cladding region and a core formed by omitting a central element and two surrounding rings of capillaries, and replacing them with a very thin-walled core tube. While the core tube approach is known to introduce additional surface mode loss resonances in the transmission [5], it provides for an increased yield, particularly useful in this study, and an easier control over the core shape during fiber drawing. The stack was used to produce a batch of structurally consistent canes from which nine fibers were produced having almost identical structure but increasing scale factor. The cladding pitch varies from $3.6 \mu \mathrm{m}$ to $7.1 \mu \mathrm{m}$ while the relative hole size, $\mathrm{d} / \Lambda$, was kept nearly constant at about $0.98 \pm 0.005$ for all fibers. The spectral attenuation of all samples was measured by using a broadband source and a standard cutback technique, where however special care was taken to excite the fundamental mode by using mode field matched launch fibers. As expected, loss peaks due to resonant coupling to surface modes were observed for all fibers, resulting in a segmentation of the photonic bandgap in two main low loss windows. In Fig. 1(a), fibers A to I, the respective regions of lowest loss (spanning from 1 to $2.3 \mu \mathrm{m}$ ) are plotted. The minimum loss of each fiber is found to follow very closely the $\lambda^{-3}$ dependence, indicating that the loss is dominated by surface scattering. The overall minimum loss $(2.5 \mathrm{~dB} / \mathrm{km})$ was measured in fibers $\mathrm{F}$ and $\mathrm{G}$ operating at $\sim 2.1 \mu \mathrm{m}$, with a $\sim 2.5 \mathrm{x}$ loss reduction as compared to fibers $\mathrm{C}$ and $\mathrm{D}$ that guide at $1.5 \mu \mathrm{m}$. Measured losses were consistent with values obtained from an accurate loss model utilizing high resolution scanning electron microscope images to calculate the fiber properties [6].

Fibers $\mathrm{H}$ and $\mathrm{I}$ have minimum loss falling beyond $2.1 \mu \mathrm{m}$ and seem to indicate an increase in the measured loss. In order to understand this, we plot the attenuation curves of two different grades -"wet" and "dry"- of silica glass 


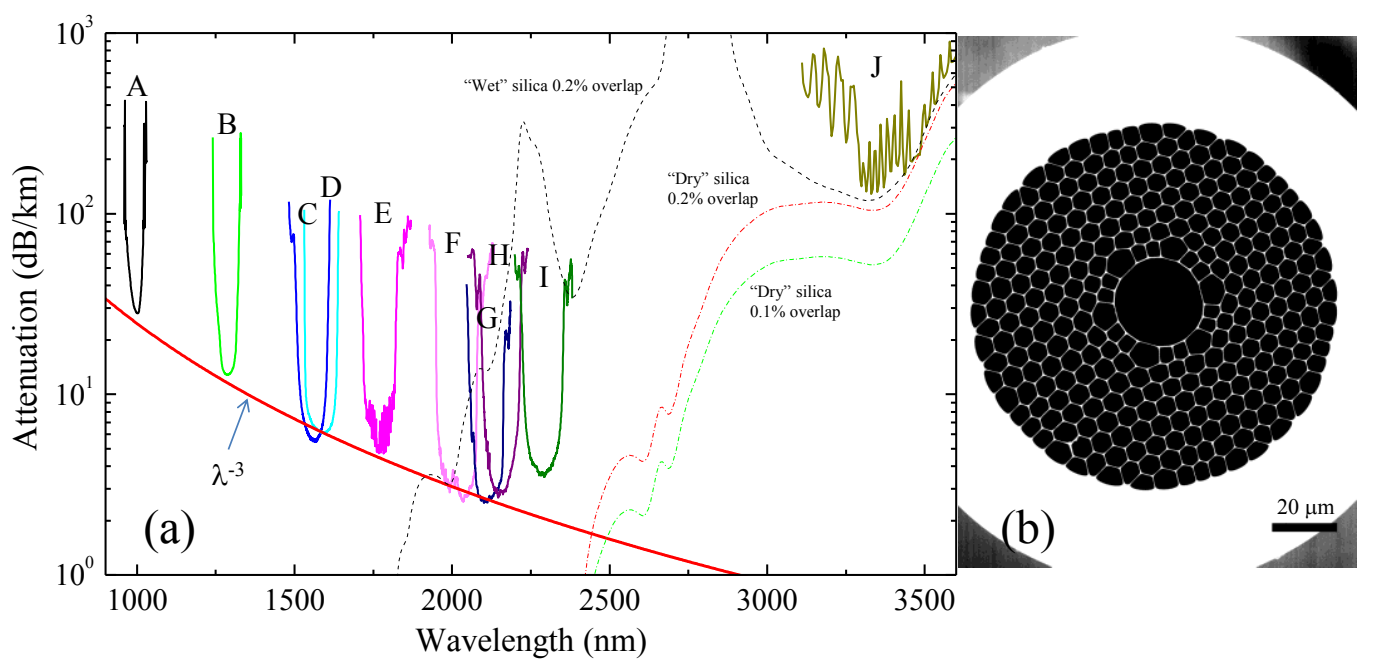

Fig. 1:(a) Scaling of minimum loss vs. wavelengths in 19 cell HC-PBGFs: different traces (labelled A to J) show the lowest loss region of ten fibers with identical structure (except for fiber J) but varying scale factor. Below $\sim 2.1 \mu \mathrm{m}$ the minimum loss follows the $\propto \lambda^{-3}$ dependence (red curve) predicted for surface scattering loss. In order to account for the loss at longer wavelengths, which is determined by the infrared absorption of silica glass, the attenuation of "wet" and "dry" silica [7] weighed by the estimated overlap factor $(0.2 \%)$ of our fibers is plotted. To provide calibration for these curves a HC-PBGF designed for operation at $3.4 \mu \mathrm{m}[8]$ is also shown (Fiber J). (b) The scanning electron microscope (SEM) image of fiber F.
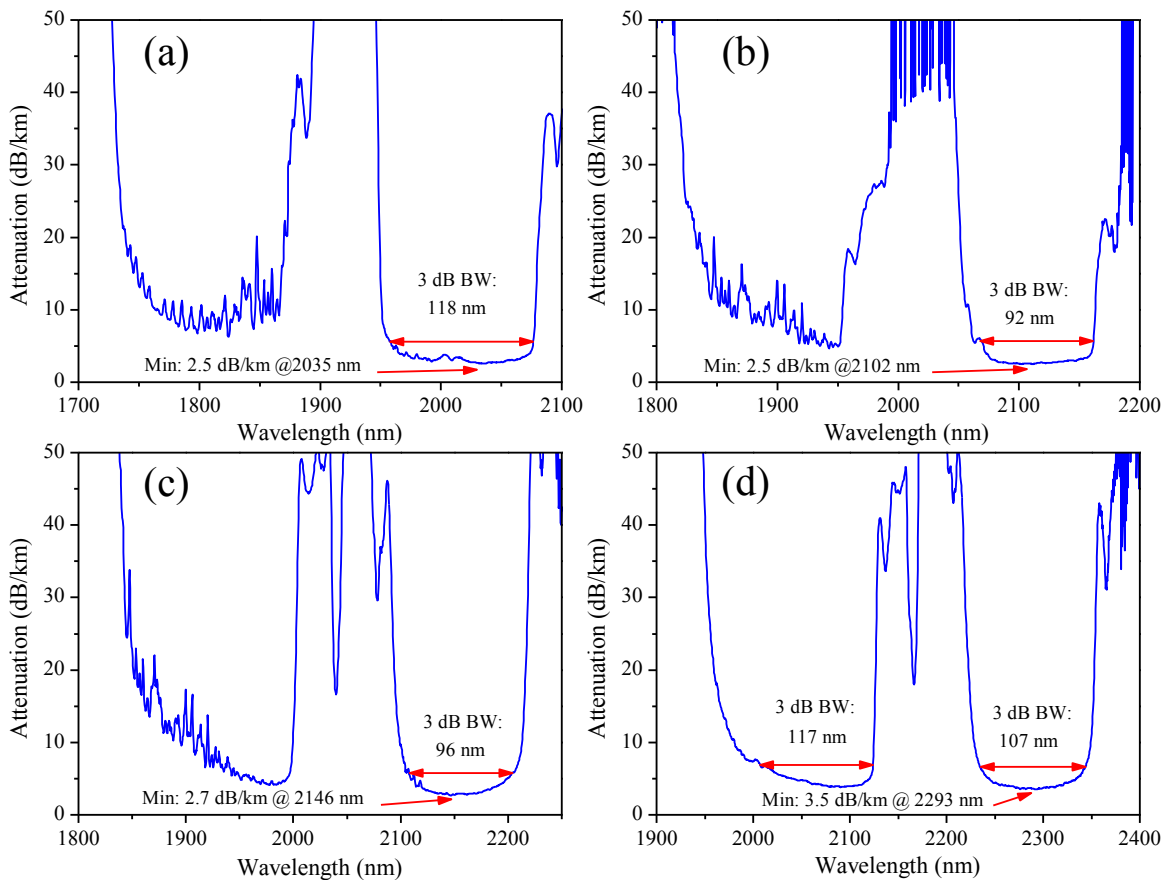

Fig. 2: Full attenuation spectra (2nm resolution) of fibers (a) F, (b) G, (c) H and (d) I, measured over 495m, $1091 \mathrm{~m}, 508 \mathrm{~m}, 493 \mathrm{~m}$ long cutbacks, respectively. Minimum loss values and $3 \mathrm{~dB}$ bandwidths are also indicated.

(which correspond to the different hydroxyl contents contained in F100 and F300 types of synthetic silica from Heraeus [7], respectively). To provide a calibration for the infrared absorption curves we also plot in Fig. 1(a) the attenuation of fiber $\mathrm{J}$, designed to operate at wavelengths around $3.4 \mu \mathrm{m}$ and characterized by a very similar mode field overlap as fibers A-I, despite having a slightly different structure [8]. Both simulations and experiments indicate that $\sim 0.2 \%$ power propagates in the glass for all fibers.

The figure shows that for a perfectly "dry" fiber the minimum absolute loss waveband would be expected to lie at wavelengths around $2.4 \mu \mathrm{m}$, much longer than the $2.1 \mu \mathrm{m}$ we measured in this experiment or the $1.9 \mu \mathrm{m}$ previously predicted [2]. This seems to suggest that although our fibers were fabricated from F300 tubes, a small but non 
negligible amount of hydroxyl ions might have been incorporated in the glass as a consequence of multiple fiber preparation processes at high temperature. Therefore, we believe that there is scope to achieve even lower attenuation values by reducing the $\mathrm{OH}$ content in the fibers and thus shifting the lowest loss region to even longer wavelengths. In addition, by using a 37 cell [9] rather than a 19 cell design would also reduce the mode field overlap with the glass to around $0.1 \%$ or lower and therefore produce a further loss reduction effect.

The full attenuation spectra of fibers F, G, H, and I are plotted in Fig. 2. Despite the presence of surface modes in the middle of the bandgap, $3 \mathrm{~dB}$ bandwidths in excess of $90 \mathrm{~nm}$ were obtained in all fibers, with that of fiber $\mathrm{F}$ as wide as $\sim 120 \mathrm{~nm}$. The lowest measured loss of $2.5 \mathrm{~dB} / \mathrm{km}$ at $2035 \mathrm{~nm}$ represents a new lowest value for HC-PBGF in this waveband and an $\mathrm{x} 2$ improvement as compared to our previous results [10]. More importantly, the attenuation values were measured over long length of bands. For example, the length of fiber $\mathrm{G}$ is $\sim 1.1 \mathrm{~km}$.

We observed substantial absorption from $\mathrm{CO}_{2}, \mathrm{H}_{2} \mathrm{O}$ and $\mathrm{HCl}$ as previously reported $[4,11]$. In an effort to remove such absorptions, we have utilized a purging method similar to the one described in [11], using ultra-dry Argon in this instance. Fig. 3 shows the attenuation of Fiber F ( $\sim 500 \mathrm{~m}$ in length) measured with an optical spectrum analyzer (OSA) with a nominal resolution of $50 \mathrm{pm}$, before and after purging for 7 days at 6 bars pressure. The three bands of $\mathrm{CO}_{2}$ at 1.95-2.1 $\mu \mathrm{m}$ were completely eliminated, providing an absorption-free low loss window. The amount of $\mathrm{HCl}$ and $\mathrm{H}_{2} \mathrm{O}$ was also very substantially decreased. Although it is preferable to remove these species during the fabrication process, it appears wholly feasible to remove absorbing gas species on fiber lengths of $\mathrm{km}$ scale.
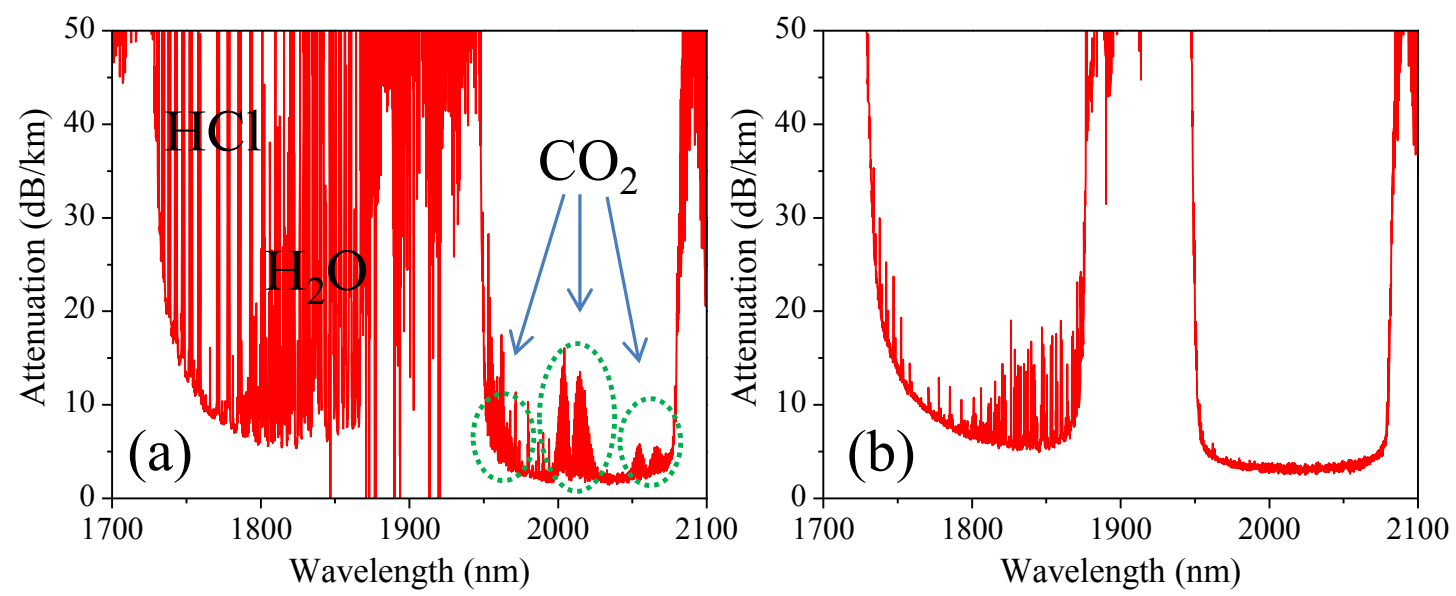

Fig. 3: High resolution attenuation spectra of fibers F, recorded (a) before and (b) after it had been purged with Argon gas for one week. The OSA resolution used was $0.05 \mathrm{~nm}$ in order to distinguish gas absorption lines.

\section{Conclusions}

We have presented for the first time the wavelength scaling of loss in 19 cell HC-PBGFs. For wavelengths shorter than $\sim 2.1 \mu \mathrm{m}$, the minimum loss is in excellent agreement with the $\lambda^{-3}$ scaling law predicted for surface scattering loss, suggesting that loss in our fibers is indeed limited by this mechanism. For longer wavelengths the loss becomes dominated by material absorption and we have identified the role of hydroxyl content in determining the precise value and wavelength of the minimum loss. Although the minimum loss region is very sensitive to the detailed fiber design, it can be deduced that this region will move to longer wavelength by reducing the overlap with silica glass and the hydroxyl content in the glass. As a part of this study, we also produced a number of HC-PBGFs with losses around $2.5-2.7 \mathrm{~dB} / \mathrm{km}$ at $2.03-2.14 \mu \mathrm{m}$, which represent a new record in this wavelength region. In addition, we have demonstrated that impact of absorbing gas species can be greatly alleviated by purging the fibers with dry gas and this approach is viable for lengths up to $500 \mathrm{~m}$, and can likely be extended to lengths of km scale.

This work was supported by FP7 (MODEGAP) and EPSRC UK through grant EP/H02607X/1

\section{References}

[1] F. Poletti, et al., Nature Photonics 7, 279-284(2013)

[2] P. J. Roberts, et al., Opt. Express 13, 236-244 (2005).

[3] E. Numkam Foukua, et al., Opt. Express 20, 20980-20991

(2012).

[4] J. K. Lyngso, et al., Opt. Express 17, 23468-23473 (2009).

[5] R. Amezcua-Correa, et al., Opt. Express 15, 17577-17586

(2007).
[6] E. Numkam Foukua, et al., Submitted to OFC 2014.

[7] O. Humbach, et al., J. Non-Cryst. Solids 203, 19-26 (1996).

[8] N. V. Wheeler et al., WSOF 2013, paper F2.36, also submitted to Opt. Lett. 2013.

[9] N. K. Baddela, et al., CLEO (2013), paper CTu2K.3.

[10] M. N. Petrovich, et al., ECOC (2012), PDP Th.3.A.5.

[11] N. V. Wheeler, et al., CLEO (2012), paper CM3N.5. 